\title{
Oesophagocardiomyotomy for Achalasia Cardia via Thoracotomy in a Teaching Hospital in Ghana: A 5-Year Retrospective Review
}

\author{
Isaac Okyere ${ }^{1 *}$, Samuel Gyasi Brenu1 ${ }^{1}$, Perditer Okyere ${ }^{2}$ \\ ${ }^{1}$ Cardiovascular and Thoracic Surgery Unit, Department of Surgery, School of Medicine and Dentistry, College of Health Sciences, \\ Kwame Nkrumah University of Science and Technology and Komfo Anokye Teaching Hospital, Kumasi, Ghana \\ ${ }^{2}$ Department of Medicine, School of Medicine and Dentistry, College of Health Sciences, Kwame Nkrumah University of Science \\ and Technology and Komfo Anokye Teaching Hospital, Kumasi, Ghana \\ Email: ^drokyere@yahoo.com
}

How to cite this paper: Okyere, I., Brenu, S.G. and Okyere, P. (2019) Oesophagocardiomyotomy for Achalasia Cardia via Thoracotomy in a Teaching Hospital in Ghana: A 5-Year Retrospective Review. Open Journal of Thoracic Surgery, 9, 31-41. https://doi.org/10.4236/ojts.2019.93004

Received: July 27, 2019

Accepted: September 23, 2019

Published: September 26, 2019

Copyright $\odot 2019$ by author(s) and Scientific Research Publishing Inc. This work is licensed under the Creative Commons Attribution International License (CC BY 4.0).

http://creativecommons.org/licenses/by/4.0/

\begin{abstract}
Achalasia cardia is a primary oesophageal motility disorder of unknown aetiology characterized manometrically by insufficient relaxation of the lower oesophageal sphincter (LES) and loss of oesophageal peristalsis; radiographically by aperistalsis, oesophageal dilation with minimal LES opening, bird-beak appearance, poor emptying of barium; and endoscopically by dilated oesophagus with retained saliva, liquid and undigested food particles in the absence of mucosal stricturing or tumour. Achalasia cardia patients usually present with difficulty in swallowing both solids and liquids and this may be associated with regurgitation, heartburn and chest pains. Treatment options include medical or pharmacologic therapy, botulinum toxin injection, pneumatic dilation and oesophagocardiomyotomy or the Heller myotomy with or without antireflux procedure and recently the POEM (Perioral oesophageal myotomy). Herein, we present our experience with four cases managed surgically via thoracotomy without antireflux surgery over a 5-year period, from January 2015 to June 2019 at the Komfo Anokye Teaching Hospital, the second largest teaching hospital in Ghana.
\end{abstract}

\section{Keywords}

Heller Myotomy, Oesophagocardiomyotomy, Thoracotomy, Achalasia Cardia

\section{Introduction}

Achalasia cardia was first described in 1674 by Sir Thomas Willis [1] as "food blockage in the oesophagus for which they were successfully managed with a di- 
lator made out the bone of a whale and sponge. However, the name achalasia, which is Greek for "failure of relaxation" was actually coined out in 1927 by Hurst who proposed organic defects in the Myenteric plexus as the cause of the failure of relaxation of the lower oesophageal sphincter [2]. Therefore, achalasia cardia is a primary oesophageal motility disorder of unknown aetiology of the oesophagus. It is characterized manometrically by insufficient relaxation of the lower oesophageal sphincter (LES) and loss of oesophageal peristalsis; radiographically by aperistalsis, oesophageal dilation with minimal LES opening, bird-beak appearance, poor emptying of barium swallow study; and endoscopically by dilated oesophagus with retained saliva, liquid and undigested food particles in the absence of mucosal stricturing or tumour". Difficulty in swallowing both solids and liquids is the main presentation and this may be associated with regurgitation, heartburn and chest pains.

An incidence of 1 in 100,000 individuals and a prevalence of 10 per 100,000 individuals have been reported by multiple articles thereby depicting its rarity [3]-[7]. A 25-year survey done in Algeria showed an incidence of 0.27 in 100,000 individuals [4]. No particular racial or sex predilection has been reported and seems to occur in all age groups but has a peak incidence of 30 - 60 years [1]. Achalasia, though rare in infants, 2 cases of symptomatic disease were reported among infants by Chatterjee et al. in 2012 [8]. Among the various options of treatment described, oesophagocardiomyotomy or Heller myotomy with antireflux procedure which can be performed via minimally invasive surgeries by laparoscopy or thoracoscopy or via open surgery through thoracotomy or laparotomy and Pneumatic Dilatation are the definitive treatment options. We present and discuss our experience with four cases managed via thoracotomy without antireflux surgery over a 5-year period, from January 2015 to June 2019 at the Komfo Anokye Teaching Hospital in Ghana.

\section{Case Histories}

\subsection{Case 1}

A 22-year-old young woman presented with progressive dysphagia to both solids and liquids for two years. This was associated with heartburn, occasional regurgitation and moderate weight loss with no other significant history and examination findings. She looked generally stable, BP of 110/78 mmHg and Heart Rate of 75 beats/minute, regular and of good volume. Abdomen was soft, flat and non-tender with normal bowel sounds. Laboratory tests were normal. The Barium swallow study as depicted in Table 1 and Figure 1, showed dilation of the entire esophagus with smooth tapering of the contrast medium at the distal third of the oesophagus at the region of the gastroesophageal junction, giving it a 'bird beak' appearance with pooling of the contrast medium proximal to the narrowing. There was incomplete relaxation of the lower esophageal sphincter with uncoordinated, non-propulsive contraction of the lower third of the esophagus and delayed emptying of contrast medium into the stomach consistent with 
Table 1. Patients clinico-demographics.

\begin{tabular}{|c|c|c|c|c|c|}
\hline Name & Age & Gender & Clinical Presentation & $\begin{array}{l}\text { Barium } \\
\text { Swallow }\end{array}$ & $\begin{array}{l}\text { Upper GI } \\
\text { Endoscopy }\end{array}$ \\
\hline Case 1 & 22 yrs. & Female & $\begin{array}{l}\text { Dysphagia to solid and } \\
\text { liquid, with heartburn } \\
\text { and regurgitation of } 2 \\
\text { years duration }\end{array}$ & $\begin{array}{l}\text { Dilated } \\
\text { esophagus with } \\
\text { smooth tapering } \\
\text { of the distal } \\
\text { oesoghagus }\end{array}$ & $\begin{array}{l}\text { Dilated } \\
\text { oesophagus } \\
\text { with retained } \\
\text { saliva, liquid } \\
\text { and } \\
\text { undigested } \\
\text { food } \\
\text { particles }\end{array}$ \\
\hline Case 2 & 31 yrs. & Female & $\begin{array}{l}\text { Dysphagia to solids and } \\
\text { liquids of } 2 \text { years. duration } \\
\text { with progressive weight } \\
\text { loss }\end{array}$ & $\sqrt{ } \sqrt{ } \sqrt{ }$ & $\sqrt{ } \sqrt{ }$ \\
\hline Case 3 & 32 yrs. & Male & $\begin{array}{l}\text { Dysphagia to solids and } \\
\text { liquids, with heart burn } \\
\text { and regurgitation for one } \\
\text { and half years }\end{array}$ & $\sqrt{ } \sqrt{ }$ & $\sqrt{ } \sqrt{ }$ \\
\hline Case 4 & 63 yrs. & Female & $\begin{array}{l}20 \text {-year history of } \\
\text { dysphagia to both solids } \\
\text { and liquid meals }\end{array}$ & $\sqrt{ } \sqrt{ }$ & $\begin{array}{l}\text { Severely } \\
\text { dilated } \\
\text { proximal } \\
\text { oesophagus } \\
\text { with food } \\
\text { debris }\end{array}$ \\
\hline
\end{tabular}

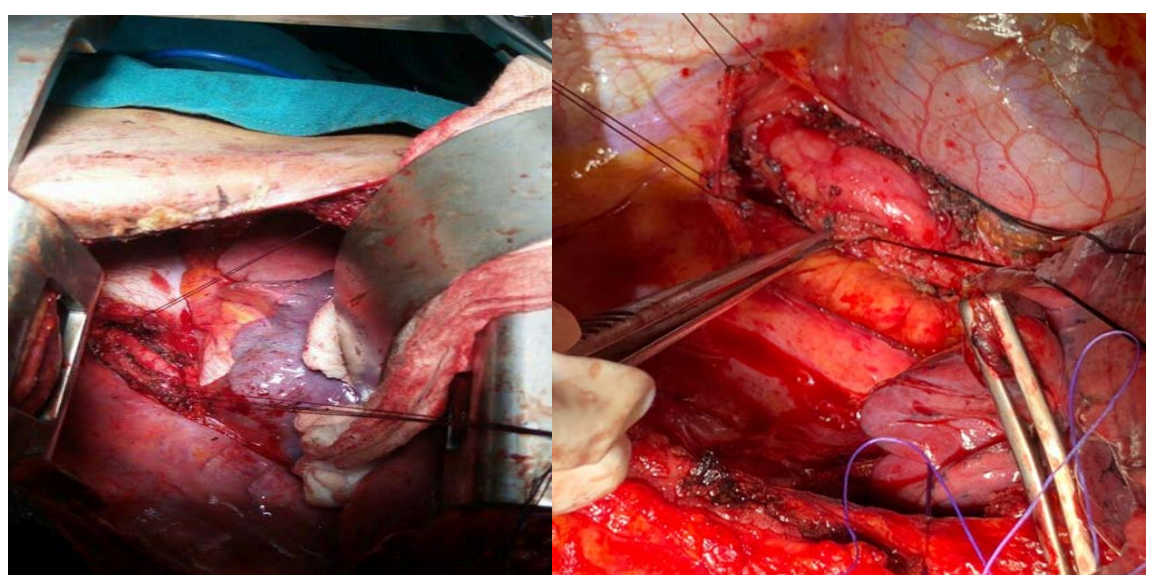

Figure 1. Intraoperative oesophageal myotomy with the exposed mucosa of cases 1 and 4 respectively.

primary achalasia cardia. Upper GI endoscopy showed a dilated esophagus with food debris. She was prepared and underwent uneventful oesophagocardiomyotomy via left thoracotomy. The post-oesophagocardiomyotomy barium swallow as shown in Figure 2, showed adequate release of the lower esophageal sphincter leading to effective drainage of contrast. She has been well without any sequelae five years after the surgery. 


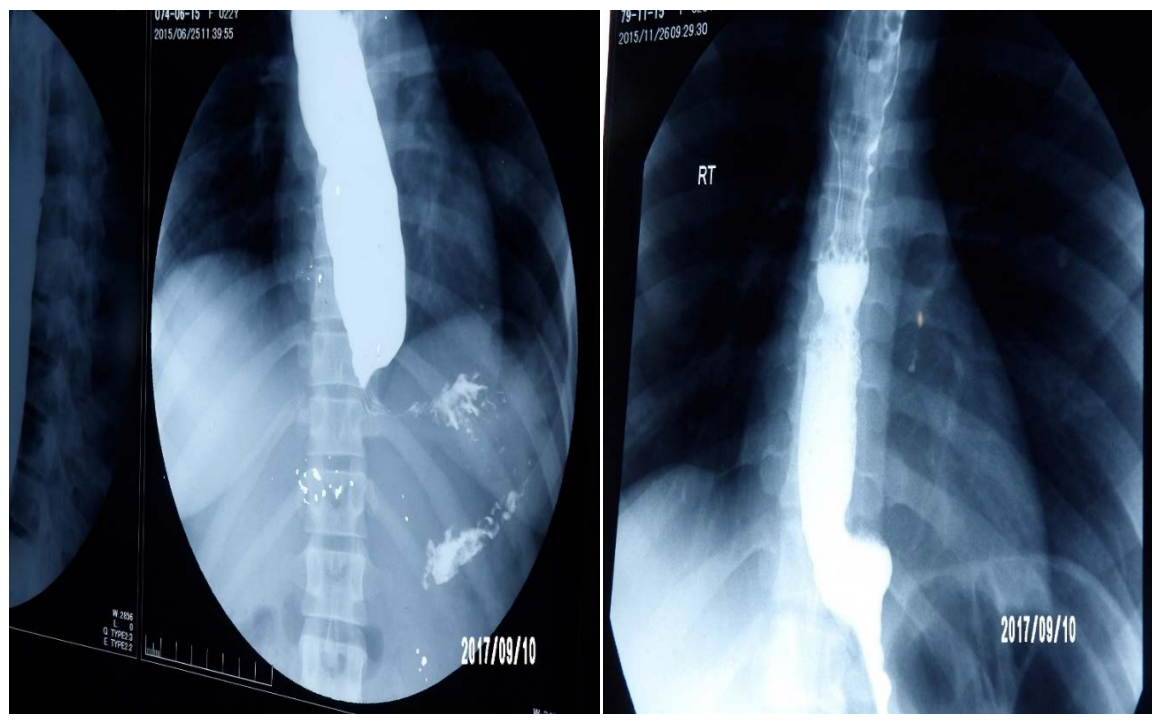

Figure 2. Preoperative and postoperative Barium study of case 1 showing the "bird's beak" appearance and the opened gastroesophageal junction post-myotomy respectively.

\subsection{Case 2}

The second case was a 31-year-old woman who also presented with a two-year history of difficulty in swallowing both solids and liquids. This was associated with heartburn, postprandial vomiting and weight loss. No significant findings on physical and laboratory examinations were noted. The Barium swallow showed a significantly dilated oesophagus throughout its length with smooth tapering of the distal oesophagus giving a "rat tail" appearance and associated prestenotic pooling of contrast medium. There was also delayed flow of contrast through the gastroesophageal junction into the stomach with no evidence of leakage, outpouching or fistulous track with the tracheobronchial system. An upper GI endoscopy showed a significant amount of food in the esophagus. She also was prepared and underwent an uneventful oesophagocardiomyotomy via left thoracotomy and has been well over four years now.

\subsection{Case 3}

A 32-year-old young man presented with difficulty in swallowing solids which have progressed to both solids and liquids for the past one and half years. This was associated with heartburn and regurgitation of previously eaten food and nocturnal cough. Other systemic reviews were normal. No significant findings in examination were noted. He had a BP of $117 / 90 \mathrm{mmHg}$, with a heart rate of 82 beats/minute, regular and of adequate volume and respiratory rate of 22 cycles/min. Labarotory investigations were normal. He also underwent an uneventful oesophagocardiomyotomy via left thoracotomy. He had been well, going on with his normal activities for the past three years with no complaints.

\subsection{Case 4}

The last case was a 63-year old known hypertensive for 32 years on Tb. Nifedi- 
pine $30 \mathrm{mg}$ daily who presented with a 20-year history of dysphagia to both solid and liquid meals. Symptoms have worsened progressively with associated repetitive stretching of the neck and frequent lubrication of food boluses with water to assists wallowing. Her dysphagia was associated with heartburn, regurgitation, weight loss, retrosternal chest pain and nocturnal dry cough but no dyspnoea or hiccups. She had been in and out of hospital for years being managed for peptic ulcer disease until 2 years prior to presenting to us when she was diagnosed with achalasia after a barium swallow study.

On examination, she was relatively stable with a BP $146 / 92 \mathrm{mmHg}$ and a heart rate of 94 beats/minute which was regular and of good volume. Abdomen was full, soft and non-tender with no palpable masses. All other organ systems were clinically normal. Accompanying investigations included oesophagogastroscopy showing dilated oesophagus with copious regurgitated fluid, gastritis with a positive $\mathrm{H}$. pyloric test. Barium swallow study showed dilated middle and distal oesophagus with retention of contrast, smooth tapered narrowing of the gastro-oesophageal junction with a thin stream of contrast noted in the distal oesophagus giving a "rat-tail" appearance, features consistent with achalasia. Abdominopelvic ultrasound did not find any pathology. Chest X-ray showed clear lung fields. Electrocardiogram was normal sinus rhythm. She also underwent uneventful oesophagocardiomyotomy via left thoracotomy and she also has been well for the past six months with no complaints.

\subsection{Surgical Approach}

All the patients underwent a standard oesophagocardiomyotomy via left thoracotomy without antireflux procedure by one surgeon. The patients were positioned on the right lateral decubitus position. Under aseptic conditions and except the case 3 who had a normal conventional endotracheal intubation, the remaining three cases underwent general anaesthesia using a size 37 FG double lumen endotracheal intubation for left lung isolation. Through the left standard posterolateral thoracotomy, the chest was entered through the $7^{\text {th }}$ intercostal space. Similar intraoperative findings were observed in all the four cases, which was mainly dilated proximal oesophagus and this was very severe in the case 4 where fibrous adhesions between the lung and chest wall, between the upper and middle lung lobes was noted as well. The rest had normal intrathoracic organs.

In all the cases, the distal oesophagus was first dissected free from the mediastinal pleura, descending thoracic aorta after freeing the inferior pulmonary ligament. It was then isolated on a sling or tape to facilitate the myotomy. As shown in Figure 1, for the cases 1 and 4, a $5 \mathrm{~cm}$ distal oesophageal myotomy as well as a $1.5 \mathrm{~cm}$ cardiomyotomy was performed with careful blunt and sharp dissection. The edges of the oesophageal myotomy were resected and this was doneto reduce the incidence of recurrence. The chest was washed with $500 \mathrm{ml}$ of warm normal saline after ensuring there is no mucosa tear and then the chest closed up in layers after inserting a size $32 \mathrm{~F}$ chest tube. All the patients were extubated on 
table. From Table 2, the average estimated blood loss was $125 \mathrm{ml}$, estimated duration of surgery was 2 hours and 7 minutes and the follow up of the patients was between six months and two and half years.

Postoperative period was uneventful for all. Patient started sips on postoperative day 5 and subsequently discharged on postoperative days 7 to 11 for follow-up on out-patient basis, one week, two weeks, one month and three months and then six months. Follow-up Barium study was done one month after the surgery.

\section{Discussion}

According to the American College of Gastroenterology, Achalasia is defined as "a primary oesophageal motility disorder of unknown aetiology characterized manometrically by insufficient relaxation of the lower oesophageal sphincter (LES) and loss of oesophageal peristalsis; radiographically by aperistalsis, oesophageal dilation with minimal LES opening, "bird-beak" appearance, poor emptying of barium; and endoscopically by dilated oesophagus with retained saliva, liquid and undigested food particles in the absence of mucosal stricturing or tumour" [4]. As reported in Table 1, all our patients were diagnosed clinically symptomatically and by the classical findings from the Barium swallow studies.

Most epidemiological surveys done have been in the western countries and an incidence of 1 in 100,000 individuals and a prevalence of 10 per 100,000 individuals have been reported by multiple studies thereby depicting its rarity [3]-[7]. We had 4 cases within five years from January 2015 to June 2019. A 25-year survey done in Algeria showed an incidence of 0.27 in 100,000 individuals [3]. No particular racial or sex predilection has been reported and seems to occur in all age groups but has a peak incidence of 30 - 60 years [4]. Even though Achalasia is rare in infants, 2 cases of symptomatic disease were reported among

Table 2. Intraoperative findings and follow-up.

\begin{tabular}{|c|c|c|c|c|c|c|}
\hline Case & Anaesthesia & $\begin{array}{l}\text { Estimated } \\
\text { Blood Loss }\end{array}$ & $\begin{array}{c}\text { Duration of } \\
\text { Surgery }\end{array}$ & $\begin{array}{l}\text { Post-Operative } \\
\text { Complication }\end{array}$ & $\begin{array}{c}\text { Follow-up } \\
\text { Barium } \\
\text { Swallow }\end{array}$ & Follow-up \\
\hline Case 1 & $\begin{array}{c}\text { Double } \\
\text { Lumen } \\
\text { intubation }\end{array}$ & $150 \mathrm{ml}$ & 2 hrs. & Nil & $\begin{array}{l}\text { Adequate } \\
\text { myotomy }\end{array}$ & $21 / 2$ years \\
\hline Case 2 & $\begin{array}{c}\text { Double } \\
\text { Lumen } \\
\text { intubation }\end{array}$ & $100 \mathrm{ml}$ & $\begin{array}{c}1 \mathrm{hr} . \\
48 \text { mins }\end{array}$ & $\mathrm{Nil}$ & $\sqrt{ } \sqrt{ }$ & 13 months \\
\hline Case 3 & $\begin{array}{c}\text { Conventional } \\
\text { anaesthesia }\end{array}$ & $150 \mathrm{ml}$ & $\begin{array}{l}2 \text { hrs. } \\
30 \text { mins }\end{array}$ & $\begin{array}{l}\text { Superficial surgical } \\
\text { site infection }\end{array}$ & $\sqrt{ } \sqrt{ }$ & 11 months \\
\hline Case 4 & $\begin{array}{c}\text { Double } \\
\text { Lumen } \\
\text { intubation }\end{array}$ & $100 \mathrm{ml}$ & $\begin{array}{l}2 \text { hrs. } \\
50 \text { mins }\end{array}$ & Nil & $\sqrt{ } \sqrt{ }$ & 6 months \\
\hline
\end{tabular}


infants by Chatterjee et al. in 2012 [8]. However, studies have shown that incidence rate tends to increase to $>10$ per 100,000 individuals in patients aged 85 90 years [9] [10].

\subsection{Aetiology and Pathophysiology}

The widely-studied and established pathophysiology underlining the development of Achalasia is the chronic and progressive degeneration of the inhibitory nerve cells in the myenteric plexus of the lower oesophageal sphincter and accompanying local and systemic inflammation with consequent loss of vasoactive intestinal peptide (VIP) and nitric oxide (NO). The resultant unopposed activity of the excitatory neurotransmitter-acetylcholine-results in failure of relaxation of the LES and aperistalsis [1] [2] [7] [9]. Histological examination of resected specimens demonstrates cytotoxic lymphocytic infiltration with evidence of complement activation within the myenteric ganglia [9].

Several studies on the aetiology of Achalasia have come up short in identifying the underlying cause of the ganglion cell degeneration in the Myenteric plexus in majority of cases [1] [2] [3] [4] [7]-[11]. Chagas disease-infection by Trypanosoma cruzi, is known to be a definitive cause of myenteric plexus ganglion cell degeneration in the LES [4]. Other conditions may mimic the clinical and manometric features of Achalasia and present as Pseudoachalasia. This accounts for $2 \%-4 \%$ of patients suspected with achalasia and associated with conditions such as tumors in the gastric cardia or those infiltrating the myenteric plexus (adenocarcinomas of the gastroesophageal junction, lung or hepatocellular carcinomas) [4] [9]. A tight fundoplication or laparoscopic adjustable gastric banding may present as Achalasia [4].

Some autoimmune diseases (Type 1 Diabetes Mellitus, hypothyroidism, Sjogren's Syndrome) [9] and viral infections (Herpes Simplex-1, Human Papillomavirus, measles) [1] [9] have been found to be more prevalent among patient with achalasia. Rarely, Achalasia has been reported to be genetically transmitted as part of clinical syndromes such as Allgrove Syndrome (also known as Triple A Syndrome), Down's Syndrome and Familial Visceral Neuropathy [4] [9].

\subsection{Clinical Presentation}

The common presenting symptoms associated with achalasia are dysphagia, regurgitation of previously eaten food, weight loss and chest pain and these happened to be present in all our patients as shown in Table 1. Dysphagia, especially to both solids and liquids, is a constant presentation in all patients with achalasia as documented in multiple reports [1] [3] [8] [9] [12] consistent with all the cases found in our study. Patients tend to alter eating habits by either eating more slowly or taking copious amounts of water with each bolus as was the typical presentation of the case 4 who was also atypically older as well. There may also be the use of manoeuvers such as arching of the back or raising the arms in an attempt to facilitate progression of bolus beyond the obstruction [12]. Other as- 
sociated symptoms such as heartburn has been reported to occur in about half of patients with achalasia and this may lead to an erroneous and common misdiagnosis of gastroesophageal reflux disease [8] [9]. In contrast, all our patients had heartburn as a symptom and the case 4 was managed for gastroesophageal reflux for a long period. Patients with substantial food accumulation in the oesophagus may also complain of respiratory complications such as aspiration and nocturnal cough [9]. In infants, the most common symptom of achalasia is vomiting of uncurdled milk, as is also seen in gastroesophageal reflux disease [8].

The non-specific nature of presentation in achalasia has been postulated to be the explanation behind the seemingly delayed diagnosis of the disease. Boeckxstaens et al. and Tebaibia et al. both reported delay of up to about 5 years until final diagnosis was made [3] [9] and our case 4 had the longest history of 20 years. In the study in Algeria by Tebaibia et al., it was noted that $5 \%$ of the patients had chest pain preceding the major symptom of dysphagia. As such, they were initially referred to a cardiologist on account of suspicion of a coronary artery disease. Also, almost half of them had significant bronchopulmonary manifestations and so were referred to a pulmonologist. These factors were mainly thought to be responsible for the delays in diagnosing achalasia cardia [3]. This was the case of our case 4 who had to be managed by many physicians for a long period before she was referred to the cardiothoracic surgeon.

\subsection{Diagnosis}

The diagnosis of achalasia includes after a classical symptomatic history, an upper GI endoscopy, a barium swallow study and if available a PH manometry. Endoscopy is done mainly to rule out a mechanical obstruction or pseudoachalasia as the cause of the presentation and this was the case for our case 4 who was older and therefore we needed to exclude pseudoachalasia. Endoscopic findings include dilated oesophagus with retained food and saliva and a tightly constricted gastroesophageal junction. Characteristic findings of a barium oesophagogram in achalasia are dilated oesophagus, aperistalsis with poor emptying of the barium and a narrow gastroesophageal junction with the "bird-beak" appearance. We employed upper GI endoscopy and Barium swallow studies for all our patients since we do not have $\mathrm{pH}$ manometry services. All the cases demonstrated the classical features in all the studies consistent with achalasia as shown in Table 1. Due to the poor sensitivity of isolated use of oesophagography and endoscopy in diagnosing achalasia, they are mostly utilized as supportive tests [4] [12]. However, in the event of classical features on both endoscopy and barium oesophagography, oesophageal manometry may be considered only as a complementary test even when the service is available [12].

Esophageal manometry is the gold standard in the diagnosis of achalasia [1]. However, due to its scarcity in low-resource countries, it is not relied on as first line in diagnosis of achalasia [3]. Criteria for diagnosis of achalasia on conven- 
tional manometry include failure of LES relaxation on swallowing and aperistalsis of the oesophageal body [11].

\subsection{Treatment Options}

Despite in-depth insight on the pathophysiology of achalasia, its aetiology has still remained a mystery. As such, approaches towards treatment are incurable but rather geared towards palliation of the associated symptoms. These therapeutic options are targeted towards reduction of LES pressure, aid oesophageal transit by relief of obstruction and enhance oesophageal emptying [1] [7] [12]. Current therapeutic options include pharmacological therapies, pneumatic dilatation and surgical myotomy. More recent and less invasive options include insertion of self-expanding metallic stents and per-oral endoscopic myotomy [1].

Main oral pharmacological drugs utilized are calcium channel blockers and nitrates. They both inhibit contraction of the LES though by different mechanisms. There are other less commonly utilized drugs such as anticholinergics, beta agonists and theophylline. Nitrates function by causing myosin light chain dephosphorylation via increasing nitric oxide concentration while calcium channel blockers inhibit cellular calcium uptake. Sublingual nifedipine is the most widely used medication for achalasia in the doses of $10-20 \mathrm{mg}$, taken $15-60$ mins before meals. It has been found to reduce LES pressure by $30 \%-60 \%$. However, side effects of hypotension, headache and dizziness from its systemic vasodilation observed in about a third of patients have limited its use. Also, there have been reports of tolerance over time [1] [9].

Currently, Sildenafil is being proposed as a possible pharmacological agent for use in achalasia due to its inhibitory effect on phosphodiesterase-5. Its action results in preservation of the nitric oxide-stimulated cyclic guanosine monophosphate and subsequent inhibition of smooth muscle contraction. However, data on its clinical use in achalasia is scanty [13].

Endoscopic injection with botulinum toxin A is another accepted pharmacological therapeutic option. It works by blocking acetylcholine release from the nerve endings. A dose of 80 - $100 \mathrm{IU}$ is injected into the LES during endoscopy and success rates of $80 \%$ within one month of injection have been reported. However, due to the rapid fading away of its effect, it is preferred, similar to nifedipine, to be for interim use while awaiting a more sustainable treatment or for high-risk patients [1] [9] [13].

Pneumatic dilatation of the LES has also been shown to be a very effective mode of treating achalasia non-surgically. Some studies have reported improving success rates with graduated dilatations using dilators of increasing sizes [7] [13]. Furuzawa-Carballeda et al. reported of success rates of $40 \%-78 \%$ and $12 \%$ $58 \%$ at 5-year and 15-year follow-ups respectively. However, this procedure is particularly associated with its most feared complication of oesophageal perforation. Rates of $<3 \%$ have been reported if dilatation is done by an experienced hand [7] [13]. GERD is seen in about a third of patients for which administra- 
tion of proton pump inhibitors are recommended [7]. None of our patients were on these medications before our evaluation, and we also did not prescribed those and rather opted for surgical intervention.

Oesophagocardiomyotomy or the Heller myotomy, the division of the circular muscle layer of the LES without disrupting the mucosa, is a procedure initially described in 1913 and has since undergone alterations to improve it [7] Heller myotomy was initially being performed via thoracotomy and subsequently via laparotomy, both with excellent outcomes in $60 \%-93 \%$ of patients but the advent of minimally-invasive surgery has seen it being replaced by thoracoscopic and currently the laparoscopic approach due to its superior visualization of the gastroesophageal junction [1] [12]. We used thoracotomy approach in all the cases probably because they were all performed by a cardiothoracic surgeon in the absence of an endoscopy suite.

The main complication associated with oesophagocardiomyotomy is the development of GERD thereby the concomitant inclusion of an anti-reflux surgery. The choice of appropriate fundoplication procedure though is still uncertain despite several studies reporting significant reduction in occurrence of GERD after myotomy with a concomittant anti-reflux surgery. Multiple studies have shown uncertainty in choosing from Dor, Toupet or Nissen fundoplication procedures in minimizing the rate of recurrence of dysphagia and regurgitation in patients who undergo myotomy for achalasia [1] [12] [13]. We did not perform any antireflux procedure, and all the patients are doing well and the case 4 was put on PPI for one month for heartburn complaints.

\section{Conclusion}

Oesophagocardiomyotomy or Heller myotomy via thoracotomy without antireflux surgery is a viable option and has the potential to give favorable results in less-resourced centres where laparoscopy or video-assisted thoracotomy surgery (VATS) suite is unavailable.

\section{Acknowledgements}

Written informed consent was obtained from the patients for publication of these case reports and accompanying images and we are grateful to the theatre staff at the Accident and Emergency centre of the Komfo Anokye Teaching Hospital.

\section{Conflicts of Interest}

None declared.

\section{References}

[1] Patel, D.A., Kim, H.P., Zifodya, J.S. and Vaezi, M.F. (2015) Idiopathic (Primary) Achalasia: A Review Rare Gastrointestinal Diseases. Orphanet Journal of Rare Diseases, 10, Article No. 89. https://doi.org/10.1186/s13023-015-0302-1 
[2] Ghoshal, U.C., Daschakraborty, S.B. and Singh, R. (2012) Pathogenesis of Achalasia Cardia. World Journal of Gastroenterology, 18, 3050-3057. https://doi.org/10.3748/wjg.v18.i24.3050

[3] Tebaibia, A., Boudjella, M.A., Boutarene, D., Benmediouni, F., Brahimi, H. and Oumnia, N. (2016) Incidence, Clinical Features and Para-Clinical Findings of Achalasia in Algeria: Experience of 25 Years. World Journal of Gastroenterology, 22, 8615-8623. https://doi.org/10.3748/wjg.v22.i38.8615

[4] Vaezi, M.F., Pandolfino, J.E. and Vela, M.F. (2013) ACG Clinical Guideline: Diagnosis and Management of Achalasia. American Journal of Gastroenterology, 108, 1238-1249. https://doi.org/10.1038/ajg.2013.196

[5] Soiza, R.L., Donaldson, A.I.C. and Myint, P.K. (2018) Vaccine against Arteriosclerosis: An Update. Therapeutic Advances in Drug Safety, 9, 259-261.

https://doi.org/10.1177/2042098618769568

[6] Aiolfi, A., Tornese, S., Bonitta, G., Cavalli, M., Rausa, E., Micheletto, G., et al. (2019) Dor versus Toupet Fundoplication after Laparoscopic Heller Myotomy: Systematic Review and Bayesian Meta-Analysis of Randomized Controlled Trials. Asian Journal of Surgery, In Press. https://doi.org/10.1016/j.asjsur.2019.03.019

[7] Furuzawa-Carballeda, J., Torres-Landa, S., Valdovinos, M.Á., Coss-Adame, E., Del Campo, L.A.M. and Torres-Villalobos, G. (2016) New Insights into the Pathophysiology of Achalasia and Implications for Future Treatment. World Journal of Gastroenterology, 22, 7892-7907. https://doi.org/10.3748/wjg.v22.i35.7892

[8] Chatterjee, S., Gajbhiye, V., De, A., Nath, S., Ghosh, D. and Das, S.K. (2012) Achalasia cardia in Infants: Report of Two Cases. IMA Journal of Management Mathematics, 44, 1-5.

[9] Boeckxstaens, G.E., Zaninotto, G. and Richter, J.E. (2017) Achalasia. Seminars in Pediatric Surgery, 26, 116-120. https://doi.org/10.1053/j.sempedsurg.2017.02.001

[10] Boeckxstaens, G.E. (2017) Revisiting Epidemiologic Features of Achalasia. Clinical Gastroenterology and Hepatology, 15, 374-375.

https://doi.org/10.1016/j.cgh.2016.11.002

[11] Chuah, S.K., Chiu, C.H., Tai, W.C., Lee, J.H., Lu, H.I., Changchien, C.S., et al. (2013) Current Status in the Treatment Options for Esophageal Achalasia. World Journal of Gastroenterology, 19, 5421-5429. https://doi.org/10.3748/wjg.v19.i33.5421

[12] Ates, F. and Vaezi, M.F. (2015) The Pathogenesis and Management of Achalasia: Current Status and Future Directions. Gut \& Liver, 9, 449-463. https://doi.org/10.5009/gnl14446

[13] Lake, J.M. and Wong, R.K.H. (2006) Review Article: The Management of Achalasia-A Comparison of Different Treatment Modalities. Alimentary Pharmacology \& Therapeutics, 24, 909-918. https://doi.org/10.1111/j.1365-2036.2006.03079.x 